\title{
Clinico-etiological Profile of Vocal Cord Paralysis
}

\author{
Shivakumar Senniappan, ${ }^{1}$ Govind Krishnan Gopalakrishnan, ${ }^{1}$ Chinnu Sudha Kumar, ${ }^{1}$ Anjali Mahendra Panicker, ${ }^{1}$ Visakh \\ Kookkal Nair, ${ }^{I}$ Shankar Radhakrishnan ${ }^{I}$
}

\begin{abstract}
Introduction
Vocal Cord Paralysis may be of central or peripheral origin based on the underlying pathology. Central Causes contribute about $10 \%$ while peripheral causes about $90 \%$ and the current study evaluated the clinical profile of vocal cord paralysis.

Materials and Methods

A cross-sectional observational study was conducted in the department of ENT for a period of one year. Patients with complaints of hoarseness or aspiration who on laryngoscopy examination with $45^{\circ}$ telescope have been diagnosed to have vocal cord paralysis were included for the study. A total of 83 patients were included in our study.

$\underline{\text { Results }}$

Vocal cord palsy was found most commonly after thyroidectomy (20.4\%) followed by the idiopathic cause (19.2\%). Certain cancers like lung cancer and neck cancers (hypopharyngeal, laryngeal and thyroid) carcinoma had also contributed significantly in the development of vocal cord palsy. Left sided vocal cord palsy (65\%) was found to be the commonest side affected followed by bilateral vocal cord palsy.

Conclusion

A proper protocol is necessary for identifying the factors responsible for vocal fold paralysis which would help in managing the condition more effectively. Before making a diagnosis as idiopathic vocal cord paralysis, detailed investigations should be carried out to rule out the possibilities of cancer, causing vocal cord paralysis.

$\underline{\text { Keywords }}$

Vocal Cord Paralysis
\end{abstract}

ABSTRACT

$\mathrm{T}$ The mechanism of production of voice which is our primary mode of communication is a combination of laryngeal, respiratory and resonance components. ${ }^{1}$ Hoarseness is an early and very important symptom of laryngeal pathology caused by simple common cold to the dreaded laryngeal malignancy. ${ }^{2}$ Voice disorders also have a significant influence on vocational, social and emotional adjustments of patients. Mostly vocal cord paralysis is considered as a sign of an underlying pathology, which may be central or peripheral in origin. Studies have shown peripheral causes $(90 \%)$ being more common than central $(10 \%))^{3}$ Vocal cord paralysis is reported commonly following surgical procedures like thyroidectomy, sometimes as a complication of intubation leading to arytenoid dislocation and rarely reported as an isolated neurologic disease. ${ }^{4}$ The cause for vocal cord paralysis usually arises in the cranial cavity, mediastinum, or neck along the course of corresponding recurrent or inferior laryngeal branch of Vagus. Due to the longer intrathoracic course of the left recurrent laryngeal nerve of left vocal cord is more common than the right. ${ }^{5}$

Hoarseness is the most common presenting symptom among patients with vocal cord palsy and other associated symptoms are stridor, breathlessness, dysphagia, sore throat, cough and haemoptysis. ${ }^{6}$ Diagnosing the cause of vocal cord palsy in the early stage is most challenging and when successful, most of the complications including death due to malignant lesions can be avoided. ${ }^{7}$ Thyroid neoplasms, lung cancer, esophageal carcinoma and mediastinal metastases are the common malignancies

1 - Department of ENT, Vinayaka Missions Kirupananda Variyar Medical College, Salem

\section{Corresponding author:}

Dr Shivakumar Senniappan

email: drshiva73@gmail.com 
which involve the recurrent laryngeal nerve leading to the paralysis of the vocal cords. Further, studies have shown that the most common cause for unilateral vocal cord palsy is bronchogenic carcinoma which involves the left vocal cord 2.5 times more than the right vocal cord. ${ }^{8}$ Anesthesia related vocal cord palsy occurs mainly during endotracheal intubation carried out for elective surgeries, leading to arytenoid dislocation. ${ }^{4} \mathrm{~A}$ viral or idiopathic vocal cord palsy generally heals without any medical intervention. All patients with vocal cord palsy need to be evaluated thoroughly with a detailed history, complete examination of head and neck, nervous system and larynx with appropriate radiological tool like chest X-ray and CT scan. MRI screening from the skull base to the diaphragm should be done to rule out the causes in the the brain stem, neck, chest and mediastinum. ${ }^{9,10}$

As of today, only very few studies had reported the clinical picture and the etiology of vocal cord paralysis and so the present study was undertaken to study the clinical profile of vocal cord paralysis.

\section{Materials and Methods}

This observational descriptive study was conducted in the department of ENT of our hospital for a period of one year between September 2016 and August 2017. The study had the clearance from the institutional ethics committee. Patients with complaints of hoarseness or aspiration, who had been diagnosed to have vocal cord paralysis on laryngoscopy with 450 telescope were included in the study and other patients with hoarseness without vocal cord paralysis were excluded. A total of 83 patients were included in this study. Informed consent was obtained from all our study subjects.

A complete history related to the causative factors of vocal cord paralysis was obtained from all the patients and a detailed physical examination was conducted. A good ENT examination including mirror examination of the larynx was done and a complete neurological examination was performed by a neurologist. All routine investigations like haemogram, urine examination, kidney function tests, liver function tests, blood glucose and serological assessment were done on all patients. Chest X-ray, and other invasive procedures like fibre- optic laryngoscopy, direct laryngoscopy with a passive mobility test, bronchoscopy, barium swallow, and if abnormal, an esophagoscopy, were performed. If all these procedures had been performed and no etiology found, a patient with vocal cord paralysis was placed in the category of "idiopathic".

The data were analyzed using SPSS version 21. Mean and standard deviation was determined for all the parametric variables and Chi-square test was used to derive statistical inference between two non-parametric variables.

\section{Results}

The minimum age in the study population was 18 years and the maximum age was 82 . Majority of the subjects in both males and females were aged above 60 years with the mean age among males was 62.6 years and among females it was 64.3 years. The ratio of females to males was 1.12. (Table I) The most common symptom presented by the patients was hoarseness, which was present among all the subjects and there were few associated symptoms like dysphagia, cough and dyspnea reported among $45 \%$ of the patients with vocal cord palsy. (Table II) Among the various etiological factors analyzed, we found that thyroidectomy was most commonly associated with vocal cord palsy (20.4\%) followed by the idiopathic cause (19.2\%). When we further analyzed the idiopathic group, we found that majority of those patients were chronic smokers. Esophagectomy and trauma to vocal cord during intubation were also found to be causes of vocal cord palsy. Certain cancers like lung cancer and neck (hypopharyngeal, laryngeal and thyroid carcinoma) also contributed significantly to the development of vocal cord palsy. (Table III) Left vocal cord palsy $(65 \%)$ was found to be the commonest, followed by bilateral vocal cord palsy $(20.4 \%)$ while involvement of the right cord $(14.4 \%)$ was found to be least common. (Table IV). Analyzing the side of vocal cord palsy and its association with various etiological factors we found a statistically significant association between these two as most of the etiological factors showed involving the left side of the vocal cord except for the neck carcinoma which had a bilateral involvement of the vocal cord. (Table V) 
Table I: Age and sex wise distribution of the study subjects

\begin{tabular}{|c|c|c|c|c|}
\hline \multirow{2}{*}{ AGE GROUP } & \multicolumn{2}{|c|}{ GENDER } & \multirow{2}{*}{ TOTAL } & \multirow{2}{*}{ P VALUE } \\
\hline & MALE & FEMALE & & \\
\hline$<\mathbf{2 0}$ & $1(2.5 \%)$ & $1(2.2 \%)$ & $2(2.4 \%)$ & \multirow{9}{*}{0.718} \\
\hline $21-30$ & $1(2.5 \%)$ & $2(4.5 \%)$ & $3(3.6 \%)$ & \\
\hline $31-40$ & $3(7.6 \%)$ & $5(11.3 \%)$ & $8(9.6 \%)$ & \\
\hline $41-50$ & $2(5.1 \%)$ & $2(4.5 \%)$ & $4(4.8 \%)$ & \\
\hline $51-60$ & $4(10.2 \%)$ & $4(9 \%)$ & $8(9.6 \%)$ & \\
\hline $61-70$ & $16(41 \%)$ & $18(40.9 \%)$ & $34(40.9 \%)$ & \\
\hline $71-80$ & $10(25.6 \%)$ & $8(18.1 \%)$ & $18(21.6 \%)$ & \\
\hline$>80$ & $2(5.1 \%)$ & $4(9 \%)$ & $6(7.2 \%)$ & \\
\hline Total & $39(100 \%)$ & $44(100 \%)$ & $83(100 \%)$ & \\
\hline Mean $\pm \mathrm{SD}$ & $62.6 \pm 11.2$ & $64.3 \pm 10.8$ & & \\
\hline
\end{tabular}

\section{Discussion}

Vocal cord paralysis is rarely caused by diseases occurring within larynx. As quoted in the literature the commonest cause for vocal cord palsy is the involvement of the recurrent laryngeal nerve mostly between the jugular foramen and its entry point into the larynx such as tumors, abscess, bulbar palsy or cerebrovascular accident. ${ }^{11}$ In our study we found majority of the patients were between 60 and 80 years of age and males and females are almost in equal numbers, whereas most of the studies had found majority of patients with vocal cord palsy in the age group of 50-60 years and males were found to be more commonly affected than females with a male: female ratio of $3: 1.12 .{ }^{13}$ In the present study we found no association between the vocal cord palsy to any particular occupation which concurred with the study done by Jayanthi et al. ${ }^{12}$ The most common symptom reported in our study was hoarseness and other symptoms like cough, dysphagia and dyspnea were related to the underlying diseases and similar observations were also made in the study conducted by Swift and Rogers. ${ }^{14}$ The onset of symptoms was sudden in $35 \%$ patients and gradual in 65\%. This is almost

Table II: Symptom wise distribution of the study subjects

\begin{tabular}{|c|c|c|}
\hline SYMPTOM & FREQUENCY & PERCENTAGE \\
\hline Hoarseness of voice & 46 & $55.4 \%$ \\
\hline Hoarseness of voice with dysphagia & 16 & $19.2 \%$ \\
\hline Hoarseness of voice with cough & 13 & $15.6 \%$ \\
\hline Hoarseness of voice with cough and dyspnoea & 8 & $9.6 \%$ \\
\hline Total & 83 & $100 \%$ \\
\hline
\end{tabular}


Table III: Distribution of the study subjects based on the various etiological factors for vocal cord palsy

\begin{tabular}{|c|c|c|}
\hline ETIOLOGICAL FACTORS & FREQUENCY & PERCENTAGE \\
\hline Post thyroid surgery & 17 & $20.4 \%$ \\
\hline Carcinoma lung & 12 & $14.4 \%$ \\
\hline Esophagectomy & 14 & $16.8 \%$ \\
\hline Idiopathic & 16 & $19.2 \%$ \\
\hline Trauma & 11 & $13.2 \%$ \\
\hline Neck carcinoma (hypopharyngeal, laryngeal and & $8.6 \%$ \\
\hline thyroid) & 8 & $2.4 \%$ \\
\hline Cardiac bypass/open heart surgery & 2 & $3.6 \%$ \\
\hline
\end{tabular}

in accordance with Swift and Rogers, who found the gradual and sudden onset of symptoms as $73.7 \%$ and $26.3 \%$ respectively. ${ }^{14}$ The paralyzed vocal cord was most commonly found in paramedian position $(70.7 \%)$ while in $18.5 \%$, paralyzed vocal cord was in intermediate position and median position was noted in $12.2 \%$ of the patients and these findings were almost at par with other studies..$^{15,16}$ In our study majority of patients showed paralysis of left vocal cord. This is in accordance with the study by Rosenthal et al. ${ }^{15}$ In the studies done by Seyed et al and Rosenthal et al, it was found that half of the patients had left vocal cord paralysis followed by right and both sides; which was almost similar to the results of our study. ${ }^{6,15}$ In the current study the most common etiology for vocal cord palsy was thyroidectomy followed by idiopathic, neoplasm and trauma. In their study, Yumoto et al had reported that surgery in $42.7 \%$, malignancy in $22.4 \%$, idiopathic in $17.4 \%$ and injuries of the neck in $2.2 \%$ of cases contributed to unilateral vocal cord paralysis vocal cord while Rosenthal et al. stated surgery in $46.3 \%$, malignancy in $13.5 \%$, idiopathic in $17.6 \%$ and neck trauma in $2.2 \%$ of subjects as reason of unilateral vocal cord paralysis in their study. ${ }^{13,15}$ In our study among the idiopathic group of vocal cord palsy we found chronic smoking as the most important factor and few of the Korean studies had also shown that chronic smoking was an important cause for vocal cord palsy. ${ }^{16-18}$ Esophagectomy (16.8\%) was found to be the third most common cause for vocal cord paralysis in our study and few studies done earlier had confirmed

Table IV: Distribution of the study subjects based on the side of vocal cord palsy

\begin{tabular}{|c|c|c|}
\hline SIDE OF VOCAL CORD PALSY & FREQUENCY & PERCENTAGE \\
\hline Left & 54 & $65 \%$ \\
\hline Right & 12 & $14.4 \%$ \\
\hline Bilateral & 17 & $20.4 \%$ \\
\hline Total & 83 & $100 \%$ \\
\hline
\end{tabular}


Table V: Association between various etiological factors and the side of vocal cord paralysis

\begin{tabular}{|c|c|c|c|c|}
\hline \multirow{2}{*}{$\begin{array}{l}\text { ETIOLOGICAL } \\
\text { FACTORS }\end{array}$} & \multicolumn{3}{|c|}{ SIDE OF VOCAL CORD PALSY } & \multirow{2}{*}{ P VALUE } \\
\hline & LEFT $(\mathbf{N}=54)$ & RIGHT (N=12) & $\begin{array}{l}\text { BILATERAL } \\
\qquad(\mathbf{N}=\mathbf{1 7})\end{array}$ & \\
\hline $\begin{array}{c}\text { Post thyroid } \\
\text { surgery }(n=17)\end{array}$ & $9(52.9 \%)$ & $5(29.4 \%)$ & $3(17.6 \%)$ & \multirow{8}{*}{$<.0001$} \\
\hline $\begin{array}{c}\text { Carcinoma lung } \\
(n=12)\end{array}$ & $11(91.6 \%)$ & $\mathbf{0}$ & $1(8.3 \%)$ & \\
\hline $\begin{array}{c}\text { Esophagectomy } \\
(n=14)\end{array}$ & $13(92.8 \%)$ & $\mathbf{0}$ & $1(7.1 \%)$ & \\
\hline Idiopathic (n=16) & $8(50 \%)$ & $4(25 \%)$ & $4(25 \%)$ & \\
\hline Trauma $(n=11)$ & $9(81.8 \%)$ & $1(9 \%)$ & $1(9 \%)$ & \\
\hline $\begin{array}{l}\text { Neck carcinoma } \\
\text { (hypopharyngeal, } \\
\text { laryngeal and } \\
\text { thyroid) }\end{array}$ & $\mathbf{0}$ & $1(12.5 \%)$ & $7(87.5 \%)$ & \\
\hline $\begin{array}{c}\text { Cardiac bypass/ } \\
\text { open heart } \\
\text { surgery }(n=2)\end{array}$ & $1(50 \%)$ & $1(50 \%)$ & $\mathbf{0}$ & \\
\hline Neurogenic $(n=3)$ & $3(100 \%)$ & $\mathbf{0}$ & $\mathbf{0}$ & \\
\hline
\end{tabular}

Chi-square value $=41.83$

esophagectomy as a cause for vocal cord palsy. ${ }^{19-21}$ In cases of malignancies or tumors the vocal cord palsy occurs either by the direct compression of the nerve by the tumor or the secondaries in the lymph nodes of neck or mediastinum having a pressure effect on the nerve. ${ }^{21}$ Various mechanical causes like aortic aneurysm, cardiomegaly, scoliosis or achalasia cardia which would compress or disturb the recurrent laryngeal nerve might lead on to vocal cord palsy. In the present study $3.6 \%$ of patients had neurogenic cause of vocal cord palsy which was almost at par with the previous studies. ${ }^{22}$

\section{Conclusion}

Voice production is a complex mechanism, involving the muscles of pharynx, palate, tongue, nose and lips. A proper protocol is necessary for identifying the factors responsible for vocal fold paralysis which would help in managing the condition more effectively. Before making a diagnosis of idiopathic vocal cord paralysis, detailed investigations should be carried out to rule out the possibilities of cancer being the etiology of vocal cord paralysis.

\section{References}

1. Samlan RA. Voice analysis. In: Cummings CW, Flint PW, Haughey BH, Robbins KT, et al editors. Cummings Otorhinolaryngology Head and Neck Surgery.4th ed. Philadelphia: Elsevier Mosby Inc; 2005.p.2008

2. Mc Glashan J. Disorders of the voice. In: Gleeson M, editor. Scott-Brown's Otorhinolaryngology Head and Neck Surgery.7th ed. London: Hodder Arnold; 2008.p.2192-4

3. Vengala RR, Kapilavaya N, Suraneni VR. Evaluation of Clinical Profile and Etiopathology for Hoarseness of Voice- A Study of 146 Cases. Int J Med Res Rev. 2015; 3(2):167-73. doi: 10.17511/ijmrr.2015.i2.030

4. Shin YH, An DA, Choi WJ, Kim YH. Unilateral vocal cord 
paralysis following a short period of endotracheal intubation anesthesia. Korean J Anesthesiol. 2013 Oct;65(4):357-8. doi: 10.4097/kjae.2013.65.4.357. PubMed PMID: 24228153; PubMed Central PMCID: PMC3822032.

5. Titche LL. Causes of recurrent laryngeal nerve nerve paralysis. Arch Otolaryngol. 1976 May; 102(5):259-61

6. Seyed Toutounchi SJ, Eydi M, Golzari SE, Ghaffari MR, Parvizian N. Vocal cord paralysis and its etiologies: a prospective study. J Cardiovasc Thorac Res. 2014;6(1):47-50. doi: 10.5681/jevtr.2014.009. Epub 2014 Mar 4. PubMed PMID: 24753832; PubMed Central PMCID: PMC3992732

7. Mau T. Diagnostic evaluation and management of hoarseness. Med Clin North Am. 2010; 94:945-60

8. Bando H, Nishio T, Bamba H, Uno T, Hisa Y. Vocal cord paralysis as a sign of chest diseases: a 15-year retrospective study. World J Surg. 2006; 30:293-8

9. Chen HC, Jen YM, Wang CH, Lee JC, Lin YS. Etiology of vocal cord paralysis. ORL J Otorhinolaryngol Relat Spec. 2007; 69:167-71

10. Glazer HS, Aronberg DJ, Lee JK, Sagel SS. Extralaryngeal causes of vocal cord paralysis: CT evaluation. AJR Am J Roentgenol 1983; 141:527-31

11. Gandhi S, Rai S, Bhowmick N. Etiological profile of unilateral vocal cord paralysis: A single institutional experience over 10 years. Journal of Laryngology and Voice 2014; 4 (2) 58-62

12. Pavithran J, Menon JR. Unilateral Vocal cord Palsy- An etiopathological study. International Journal of Phonosurgery and Laryngology 2011; 1(1):5-10

13. Yumoto E, Minoda R, Hyodo M, Yamagata T. Causes of recurrent laryngeal nerve paralysis. Auris Nasus Larynx 2002; 29:41-5
14. Swift AC, Rogers J. Vocal cord paralysis in children. J Laryngol Otol. 1987; 101(2):169-71. DOI: https://doi.org/10.1017/ S0022215100101446

15. Rosenthal L H, Benninger $M$ S, Deeb $R \mathrm{H}$. Vocal fold immobility: A longitudinal analysis of etiology over 20 years. Laryngoscope 2007; 117:1864-70

16. Byeon $\mathrm{H}$. The association between lifetime cigarette smoking and dysphonia in the Korean general population: findings from a national survey. Peer J 2015; 3:e912; DOI 10.7717/peerj.912

17. Awan SN. The effect of smoking on the dysphonia severity index in females. Folia Phoniatrica et Logopaedica 2011; 63:65-71 DOI 10.1159/000316142

18. Byeon $\mathrm{H}$. The association between smoking and self-reported voice problems in the Korean adult population. Journal of Speech-Language \& Hearing Disorders 2011; 20:17-30 DOI 10.15724/jslhd.2011.20.3.002

19. Scholtemeijer MG, Seesing MFJ, Brenkman HJF, Janssen LM, van Hillegersberg $\mathrm{R}$ et al. Recurrent laryngeal nerve injury after esophagectomy for esophageal cancer: incidence, management, and impact on short-and long-term outcomes. J Thorac Dis. 2017; 9(Suppl 8):S868-78

20. Gelpke H, Grieder F, Decurtins M, et al. Recurrent laryngeal nerve monitoring during esophagectomy and mediastinal lymph node dissection. World J Surg. 2010; 34:2379-82

21. Koyanagi K, Igaki H, Iwabu J, et al. Recurrent laryngeal nerve paralysis after esophagectomy: Respiratory complications and role of nerve reconstruction. Tohoku J Exp Med. 2015; 237:1-8

22. Ahmad S, Muzamil A, Lateef M. A Study of incidence and etiopathology of vocal cord paralysis. Indian J Otolaryngol Head Neck Surg. 2002; 54(4); 294-96. 